\title{
LOS USOS DE LAS TIC EN LAS AULAS COMO FACTOR PREDICTIVO DEL ESTADO EMOCIONAL DE LOS ESTUDIANTES*
}

\author{
Pilar Colás-Bravo** \\ Salvador Reyes-de Cózar \\ Jesús Conde-Jiménez \\ Universidad de Sevilla
}

\section{RESUMEN}

El objetivo de esta investigación es identificar la capacidad predictiva sobre el estado emocional que tiene el uso de las TIC en las aulas. La muestra se compone de 1982 sujetos pertenecientes a centros escolares andaluces de larga experiencia en la implantación y uso de las TIC. Para el análisis de los datos se realiza una Regresión Logística Binaria. Los resultados confirman la fiabilidad de las escalas, así como como la bondad de ajuste de nuestro modelo propuesto. Los resultados confirman que los estudiantes presentan niveles más altos de emociones positivas cuando los usos y aplicaciones de las TIC son más complejos. Se concluye que cuando los estudiantes son agentes activos y creadores de sus propios contenidos, tienen una mejor predisposición hacia el aprendizaje y presentan mejor estado emocional. Palabras Clave: estado emocional, TIC, modelos predictivos, educación primaria, educación secundaria.

\section{USES OF ICT IN CLASSROOMS AS A PREDICTIVE FACTOR OF STUDENTS' EMOTIONAL STATE}

\section{Abstract}

The objective of this research is to identify the predictive capacity on the emotional state that has the use of ICT in the classrooms. The sample is composed of 1982 subjects belonging to Andalusian school centers with long experience in the implementation and use of ICT. For the analysis of the data, a Binary Logistic Regression is performed. The results confirm the reliability of the scales as well as the goodness of fit of our proposed model. The results confirm that students have higher levels of positive emotions when the uses and applications of ICTs are more complex. It is concluded that when students are active agents and creators of their own content, they have a better predisposition towards learning and have a better emotional state.

KEYWORDs: emotional state, ICT, predictive models, primary school, secondary school.

DOI: http://doi.org/10.25145/j.qurricul.2018.31.001 


\section{INTRODUCCIÓN}

La alfabetización digital es uno de los retos que se plantean los sistemas educativos en la actualidad. En este sentido, las políticas educativas han hecho una apuesta decidida para su consecución, a través del fomento del uso de las TIC en la actividad escolar. Sumado a lo anterior, la acción política VII Programa Marco de Investigación, Desarrollo Tecnológico e Innovación de la Unión Europea -Horizonte 2020-, junto con la Agenda Digital para Europa o i2020 (Comisión Europea, 2010), pone sobre la mesa la necesidad de que los estudiantes, futuros ciudadanos, desarrollen la correspondiente alfabetización digital que les permita ser aprendices autónomos y conectados positivamente con los retos y exigencias que demandan los contextos educativos y laborales. En otras palabras, que adquieran y sean capaces de poner en práctica competencias digitales que les permitan un desarrollo personal y social acorde con la cultura y modelos sociales actuales, dinámicos y en continuo cambio (Johnson, Adams Becker, Estrada \& Freeman, 2014; OCDE, 2015).

La alfabetización digital se relaciona de forma directa con la competencia digital (Martin \& Grudziecki, 2006), evidenciada explícitamente mediante los usos de las TIC que desarrollan las personas (De Pablos, Colás, Conde \& Reyes, 2017), que constituye una de las ocho capacidades clave para poder participar en el aprendizaje a lo largo de la vida (Comisión Europea, 2006). Estos conocimientos, destrezas y/o habilidades digitales son necesarios de cara a garantizar a la ciudadanía su participación activa en la sociedad del siglo Xxi (Senkbeil, Ihme \& Wittwer, 2013; Zhou, 2013)

Sin embargo, el grueso de los estudios sobre alfabetización digital se centran, básicamente, sobre los usos de herramientas tecnológicas (van Deursen \& van Dijk, 2009; Zylka, Christoph, Kroehne, Hartig \& Goldhammer, 2015). Este campo de estudio, recientemente, se está nutriendo de la incorporación y desarrollo de la dimensión emocional, dando como resultado la necesidad de incorporar ésta para el entendimiento

* Los datos recogidos en este artículo forman parte del Proyecto de I+D del Ministerio de Ciencia e Innovación titulado Las políticas de un «ordenador por niño» en España, visiones y prácticas del profesorado ante el programa Escuela 2.0. Un análisis comparado entre comunidades autónomas (Ref: CS02010), aprobado dentro del Plan Nacional de I+D+i 2008-2011. El programa Escuela 2.0 es suprimido en 2012, tres años después de su puesta en marcha; por esta razón se incorpora un nuevo objetivo científico, no previsto inicialmente: conocer las aplicaciones que se hacen de las TIC en las aulas en las Comunidades Autónomas una vez suprimido el Programa Escuela 2.0. Los datos utilizados en esta aportación corresponden a esta última fase del proyecto de investigación. En este artículo se han utilizado los términos en su género masculino, con valor sintético y genérico, en lugar de la dualidad masculino/femenino. Este uso no supone discriminación sexista alguna, sino un intento de no realizar una escritura demasiado extensa y proporcionar mayor fluidez y claridad al texto.

** Los autores de esta aportación son miembros del Grupo Investigación, Evaluación y Tecnología Educativa (GIETE.HUM-154/http://giete.us.es) y pertenecen a REUNI+D (Red Universitaria de Investigación e Innovación educativa. Cambios sociales y retos para la educación en la era digital. MINECO. EDU201454943REDT: http://reunid.eu). Universidad de Sevilla. C/ Pirotecnia, s/n. 41013 Sevilla (España). 
de la alfabetización digital (Chen \& Sun, 2012; De Pablos, Colás, González \& Camacho, 2013; Wilson, Scalise \& Gochyyev, 2015). Esta perspectiva de investigación emergente se sustenta en estudios sobre nuevas alfabetizaciones (New Literacies Studies) que, influenciados por el enfoque sociocultural, incorporan al constructo de alfabetización digital una dimensión emocional vinculada a su desarrollo (Area \& Ribeiro, 2012; Aviram \& Eshet-Alkalai, 2006; Colás, Conde \& González, 2015).

La investigación sobre competencia digital ha generado perspectivas y enfoques diversos, incluyendo análisis tanto de naturaleza social como de índole emocional (Ilomäki, Kantosalo \& Lakkala, 2011). La perspectiva social queda ilustrada en los estudios sobre brecha digital, que muestran cómo la desigualdad de acceso a los medios y recursos tecnológicos genera diferencias en cuanto a los niveles de logro de competenciales o habilidades digitales (Conde, 2017). Por otra parte, se observa otra destacada línea de investigación sobre la actitud de los sujetos hacia las tecnologías, desarrollándose conceptos ampliamente difundidos como la tecnofobia y la tecnofilia. La tecnofobia es un miedo o aversión hacia las tecnologías o dispositivos complejos, mientras que la tecnofilia es lo opuesto, una pasión o disfrute por la tecnología. Estos conceptos llevan implícitos un factor emocional que condiciona la actitud y el uso de las mismas y, en consecuencia, el desarrollo de diferentes niveles competenciales.

A pesar del interés científico y lo novedoso de estos enfoques, en la actualidad no existe una línea de investigación consolidada, de carácter empírico, que relacione el uso de las TIC con la dimensión emocional en los contextos educativos. Por esta razón, este trabajo pretende contrastar, empíricamente, un modelo en el que se relacionan los usos tecnológicos que se producen en los centros escolares y el estado emocional asociado a los mismos.

\section{LA INVESTIGACIÓN SOBRE LAS EMOCIONES ASOCIADAS AL USO DE LAS TIC}

Tal como se ha planteado, en la actualidad, asistimos a una incorporación progresiva de los aspectos emocionales en estudios vinculados a la alfabetización digital, como consecuencia de la relevancia adquirida por la dimensión afectiva como objeto de estudio de numerosas ramas del conocimiento (Serrano, 2015). Las emociones son un aspecto fundamental de la experiencia humana (Kwah, Milnes, Tsai, Goldman \& Plass, 2016) y, por esta razón, los investigadores empiezan a señalar la importancia de éstas como factor clave en los procesos cognitivos y de aprendizaje (Park, Plass $\&$ Brüenken 2014). En esta línea, estudios como el de Arnold (2000) o, más recientemente, el de Rodríguez (2016), proponen que las emociones son un medio que puede ayudar al fomento, bloqueo o mantenimiento de estos procesos. Para Olitsky \& Milne (2012), el concebir a los estudiantes como agentes activos en sus prácticas de aprendizaje implicaría prestar atención a las emociones asociadas a las mismas. Esto es, las emociones de los estudiantes son las que guían las necesidades y metas que tenemos que cubrir en las aulas (Kwah, Milnes, Tsai, Goldman \& Plass, 2016).

Sin embargo, a pesar de la relevancia de investigar las emociones asociadas al uso de las TIC, existe poca investigación empírica que respalde estos supuestos 
dentro del ámbito educativo. Así, como apuntan Bhandari \& Chang (2014), los estudios se han centrado, principalmente, en abordar los usos instrumentales de las tecnologías digitales, dejando de lado la faceta emocional asociada a los mismos. En esta misma línea, investigadores como De Lera, Fernández \& Almirall (2009) y Wang (2014) consideran que son necesarias más investigaciones para identificar las emociones experimentadas cuando se aplican y se usan las TIC en la enseñanza.

No obstante, existe una línea de investigación emergente, en el ámbito educativo, que está originando estudios que vinculan los usos de las TIC a las emociones derivadas de los mismos. En este sentido, entendemos conveniente hacer una revisión científica de las aportaciones de los mismos, cara a sistematizar los principales núcleos de interés en los que se está proyectando esta línea.

La atención a la diversidad es una de las áreas educativas en las que se aprecia un especial interés por vincular los usos de las tecnologías con la dimensión emocional. En este sentido, se ha encontrado un primer bloque de estudios que señalan el papel mediador de las TIC para el desarrollo personal en la enseñanza de sujetos con trastorno del espectro autista (Lozano, Ballesta \& Alcaraz, 2011; García, Garrote \& Jiménez, 2016). Estos estudios demuestran que los autistas manifiestan una buena predisposición a trabajar con las tecnologías digitales (Moore \& Taylor, 2000; Hardy, Ogden, Newman \& Cooper, 2002; Terrazas, Sánchez \& Becerra, 2016). Así, han surgido estudios que orientan el diseño de software y aplicaciones tecnológicas específicas a través de las que fomentar la dimensión emocional de los autistas (Charitaki, 2015).

Otros estudios, por su parte, están enfocados en explorar la correlación existente entre el uso de diferentes materiales multimedia, los estados emocionales de los alumnos y el rendimiento escolar (Shen, Wang \& Shen, 2009; Chen \& Lee, 2011; Chen \& Wang, 2011; Um, Plass, Hayward \& Homer, 2011; Bi \& Fan, 2011; Chen \& Sun, 2012). Además, los investigadores se centran en indagar variables personales y sus posibles efectos causales en los usos de las TIC y sus emociones asociadas. En concreto, Chen \& Wang (2011) especifican que las emociones dependen del género, comprobándose que existe una diferencia significativa entre los sexos en los estados emocionales y su relación con el uso que los estudiantes hacen de las tecnologías digitales.

Otro foco de atención científica se orienta sobre las emociones que se producen por el uso de las TIC como herramientas para la comunicación (Belli, Harré \& Íñiguez, 2014; Belli \& Gil-Juárez, 2011), debido a la relevancia lograda por las TIC en el plano de la interacción social (Serrano, 2015). Del mismo modo, Suryani, Subali \& Ratu (2016) examinan cómo la interacción mediada por las TIC y su contexto de comunicación pueden afectar el comportamiento de los docentes, y consideran que la forma en la que los profesores sienten, piensan y responden a los mensajes mediados por tecnología de sus estudiantes orienta el proceso de aprendizaje, ya que se desarrolla y/o fomenta un tipo determinado de uso y/o aplicación de las tecnologías digitales.

Un núcleo importante de investigaciones se centra en el análisis de la naturaleza de las emociones en contextos educativos enriquecidos con TIC (Butz, Stupnisky \& Pekrun, 2015). Así, Valverde, Fernández \& Revuelta (2013) señalan que cuando 
se hace un uso educativo de las tecnologías digitales se producen, principalmente, emociones positivas como felicidad, orgullo, satisfacción, mejora de la autoestima y de la autoconfianza, así como una mejora en la percepción de la eficacia de la actividad realizada con las TIC. Igualmente, Nummenmaa (2007) y Wang (2014) encontraron que un mejor dominio y uso de las tecnologías permite a los estudiantes una mejor relación con estas herramientas, lo que a su vez produce emociones positivas. Por el contrario, según los mismos autores, aquellos estudiantes que no hacen un uso eficaz de las TIC experimentan emociones negativas y se vuelven ineficientes en su aprendizaje en un entorno de aprendizaje mediado por las TIC. Igualmente, Zhou (2013) examinó la relación entre los estados emocionales de los estudiantes y su capacidad para la aplicación de las TIC con fines informacionales, comprobando que aquéllos que presentan emociones positivas, en comparación con los grupos de estudiantes con emociones negativas o mixtas, se caracterizaron como más eficientes en el uso de las mismas. Sin embargo, estudios como el de Marchand \& Gutiérrez (2012), quienes analizaron el sentimiento de frustración en cursos universitarios, tanto en línea como presenciales y el de Lehman, D’Mello \& Graesser (2012), quienes estudiaron las emociones vinculadas a sistemas virtuales de tutoría, concluyen, de forma opuesta, que los estudiantes presentaban mayores niveles de emociones negativas en entornos de aprendizaje en los que están presenten las tecnologías.

Por último, podemos referenciar investigaciones sobre el uso de las TIC y su relación positiva con variables subjetivas tales como bienestar, engagement y motivación. En esta línea, el informe Digital for Europe (Comisión Europea, 2014) indica que las TIC tienen un papel de gran peso en nuestras vidas privadas que va más allá del ocio y de las relaciones sociales, dado que las prácticas de uso de las TIC se relacionan con el bienestar. También De Pablos, Colás, González \& Camacho (2013) confirmaron la relación existente entre el bienestar subjetivo y la innovación docente con TIC. Esto coincide con el trabajo de Sekret \& Kommers (2014), quienes relacionan la competencia digital como clave para el bienestar, o los de Schmidt, Kackar-Cam, Strati \& Shumow (2015) y Reyes (2016), que relacionan los niveles de engagement con la percepción del sentimiento de competencia, así como el de Zhong (2011), que se interesa por identificar la influencia de factores subjetivos en el dominio competencial. Finalmente, existen estudios que apuntan la relación existente entre el uso de las TIC y la motivación para el aprendizaje (Huertas \& Pantoja, 2016; Kaiser Family Fundation, 2010; Eow, Wan-Zah, Rosnaini \& Roselan, 2010; Losada, Correa \& Fernández, 2017; Méndez, 2015; Zylka, Christoph, Kroehne, Hartig \& Goldhammer, 2015). Todos estos estudios, por tanto, revelan la relación existente entre dimensiones emocionales subjetivas y el uso de las tecnologías digitales.

Tal como se ha visto, aunque están apareciendo estudios que relacionan el uso de las tecnologías digitales y la dimensión afectiva de las personas, se ha detectado una laguna en cuanto a la existencia o aportación de modelos teóricos que sustenten empíricamente estos supuestos. Por ello, es necesario que surjan nuevas investigaciones empíricas que vayan enriqueciendo el corpus de conocimiento de esta línea de investigación emergente. Por esta razón, este estudio pretende estudiar la capacidad predictiva de los niveles de competencia digital de los estudiantes en los usos de las TIC, sobre sus emociones, en contextos educativos. 


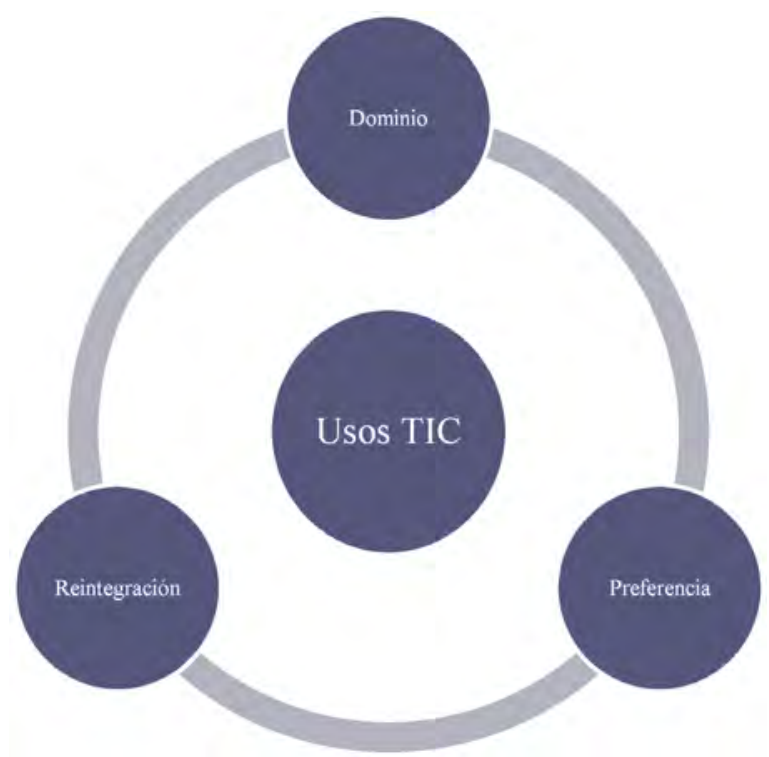

Figura 1. Categorías asociadas a la dimensión Usos de las TIC en contextos educativos.

\section{MODELO PREDICTIVO DE LOS EFECTOS DEL USO DE LAS TIC EN LOS CONTEXTOS EDUCATIVOS SOBRE EL ESTADO EMOCIONAL DE LOS ESTUDIANTES}

Tal como se ha visto, la dimensión afectiva se considera un factor clave en las decisiones y comportamientos humanos dentro de muchos contextos sociales. En los contextos educativos, concretamente, cada vez son más los estudios que empiezan a poner el foco en la relación existente entre las tecnologías digitales y las emociones asociadas a su uso. Sin embargo, pocos de estos estudios toman enfoques sistemáticos, lo que está generando conclusiones inconsistentes y, en algunos casos, recomendaciones contradictorias para investigadores y profesionales. Por este motivo, para este estudio se ha tomado como punto de partida la sistematización de la dimensión emocional y su relación con las TIC propuesta por Zhang (2013), con el objetivo de elaborar una conceptualización lo más rigurosa posible y que sirva de base teórica para futuras investigaciones.

El modelo que aquí se propone tiene como objetivo evaluar las emociones que experimentan los estudiantes cuando desarrollan actividades escolares mediante las TIC. Consideramos que detectar los efectos emocionales asociados a los usos nos permitiría obtener evidencias sobre las interacciones de aprendizaje de los estudiantes con las TIC, haciendo, además, que estas emociones sean explícitas para ellos. De acuerdo con Arguedas, Daradoumis \& Xhafa (2016), esta evaluación aportaría a los estudiantes una propia conciencia emocional, que supondría la base a la hora de 


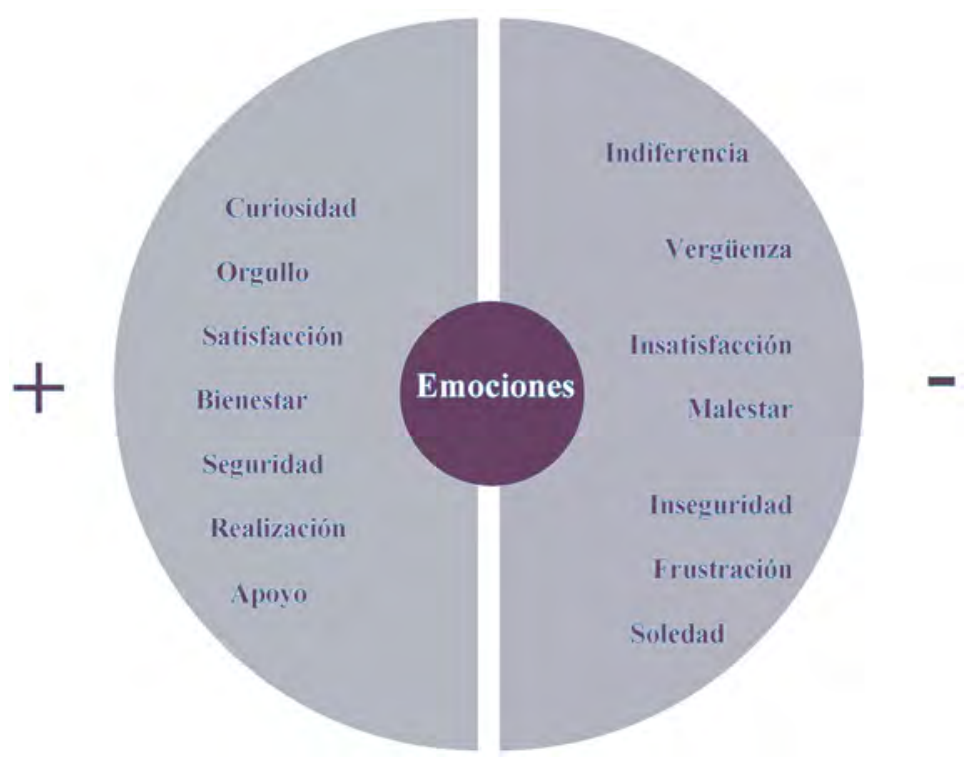

Figura 2. Listado de emociones asociadas al uso de las TIC.

orientar la toma de decisiones sobre los usos de las TIC. Para los autores, desarrollar una conciencia emocional tiene un impacto positivo inmediato en los niveles de engagement de los estudiantes, dando como resultado una mejora en las prácticas de aprendizaje.

El modelo que se presenta consta de dos dimensiones. Por un lado, se propone como variable independiente los usos de las TIC en los contextos educativos y, por otro lado, la variable dependiente la constituyen las emociones asociadas a los mismos.

En cuanto a la dimensión Usos TIC, ésta se ha concretado a través de tres niveles distintos a los que hemos denominado Dominio, Preferencia y Reintegración, basándonos en los constructos del enfoque sociocultural. Por Dominio se entienden aquellos usos instrumentales o básicos de las TIC que realizan los estudiantes. En otras palabras, usos que implican la aplicación de destrezas vinculadas al acceso y manejo de las TIC a nivel de usuario (De Pablos, Rebollo \& Aires, 1999; Colás, Rodríguez \& Jiménez, 2005). Con Preferencia se hace referencia a cuando los estudiantes prefieren el uso de las TIC, y no otras herramientas, para resolver las tareas escolares (Colás, 2006). Por su parte, Reintegración hace referencia a aquéllos usos que hacen los estudiantes de las TIC para fines distintos de aquellos para los que los han aprendido (De Pablos, 2003; Conde, 2017). Esto es, los usos TIC hacen referencia a conductas manifiestas o explícitas en las que se evidencian estos constructos socioculturales internos.

En cuanto a la dimensión Emociones, nos hemos basado en un metaanálisis sobre estudios de los constructos afectivos vinculados a las TIC realizado por Zhang 


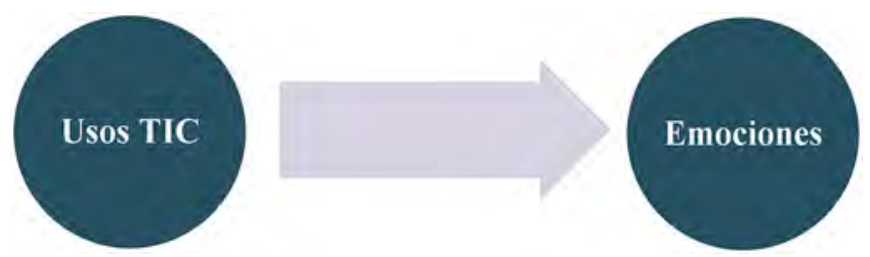

Figura 3. Modelo de relación entre los usos TIC y emociones asociadas.

(2013). Partiendo de esta sistematización, se ha creado una nueva categorización de emociones agrupándolas en dos grandes bloques: emociones positivas y emociones negativas. Según Beaudry \& Pinsonneault (2010), las emociones se definen como un estado mental en respuesta a la evaluación de los usos de las TIC, y que orientan futuras acciones. Según varios autores, esta evaluación puede generar emociones positivas (felicidad, orgullo, bienestar, satisfacción, diversión...) o negativas (enojo, ansiedad, frustración, insatisfacción, etc.) (Parboteeah, Valacich \& Wells, 2009; Thong, Hong \& Tam, 2006; van der Heijden 2004; Venkatesh \& Bala 2008).

Finalmente, nuestro modelo establece una relación causal entre ambas dimensiones, es decir, las emociones son resultado directo de los usos. Tal como se ha visto, esta relación causal tiene su fundamentación teórica en los estudios expuestos en los que se relacionan estas dos dimensiones.

\section{MÉTODO}

El objetivo principal de esta investigación es el de determinar el efecto de los usos de las TIC en los contextos educativos sobre el estado emocional de los estudiantes. Para satisfacer este objetivo general, se pretende determinar, empíricamente, la capacidad predictiva del modelo elaborado y planteado en la parte teórica, determinando, además, los pesos individuales de las diferentes variables independientes (Niveles de Usos de las TIC: Domino, Preferencia y Reintegración) sobre la variable dependiente (Estado emocional).

\section{DISEÑO DE LA INVESTIGACIÓN}

Selección De LA MUeSTRA

La población objeto de estudio se compone de 291551 estudiantes matriculados en centros de Primaria y Secundaria de la provincia de Sevilla. Se realiza un muestreo por conglomerados, siendo la unidad muestral los centros que han participado de forma continuada en los programas de políticas TIC dirigidos a la integración de las TIC en los centros escolares. La muestra final se compone de 


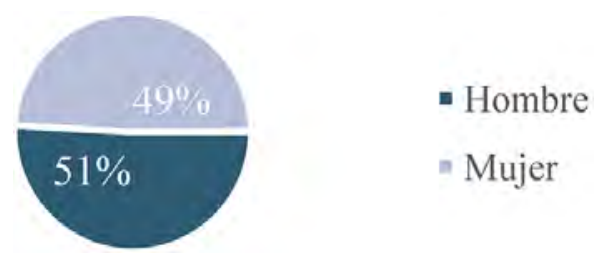

Figura 4. Distribución de la muestra por sexo.
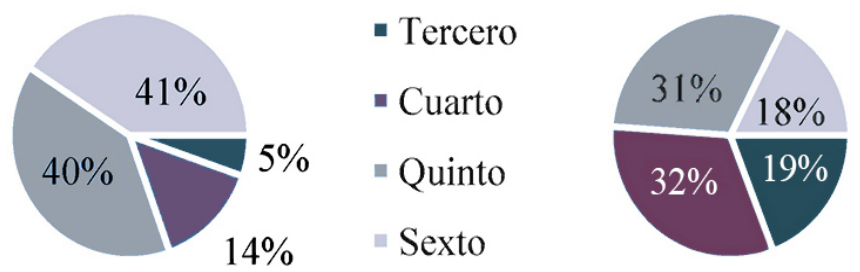

- Primero

- Segundo

- Tercero

Cuarto

Figura 5. Distribución de la muestra por áreas Nivel Educativo y Curso (Primaria/Secundaria).

un total de 1982 estudiantes, siendo representativa de la población con un nivel de confianza del 99\%, y con un error muestral del 3\%.

La distribución de la muestra por sexos es del 50,8\% alumnos y 49,2\% alumnas. En cuanto al nivel educativo, el 46,9\% es alumnado de Educación Primaria y el 53,1\% alumnado de Secundaria. En Educación Primaria la distribución por cursos es la siguiente: 5,5\% Tercero, $14,7 \%$, Cuarto, 39,4\%, Quinto y 40,4\% Sexto; mientras que en Secundaria: 19,2\% Primero, 32,2\% Segundo, 31,1\% Tercero y $17,3 \%$ Cuarto.

\section{TÉCNICA DE RECOGIDA DE DATOS Y DISEÑO DEL INSTRUMENTO}

La recogida de datos de las variables de estudio se lleva a cabo en base a un cuestionario construido y elaborado ad hoc a través de dos escalas, basándonos en la categorización realizada para cada una de las dos dimensiones planteadas en el modelo expuesto en el marco teórico. Concretamente, se diseńan dos escalas tipo Likert de 1 a 5 (siendo 1 la valoración más baja y 5 la más alta) con el propósito de conocer los usos que realiza el alumnado de las TIC y los efectos de estos usos sobre su estado emocional. Además, se realiza el análisis de la validez y de la consistencia interna de las escalas.

Con respecto al diseño de los ítems que componen las escalas, en el caso de la primera: Uso de las TIC en las aulas, se incluyen 11 ítems tal como muestra la tabla 1. 


\begin{tabular}{ll}
$\mathrm{D}_{1}$ & 1. Realización, sin problemas, de actividades escolares con el ordenador \\
$\mathrm{D}_{2}$ & 2. Conocimiento del funcionamiento de las tecnologías, y su uso para hacer los deberes \\
\hline $\mathrm{D}_{3}$ & 3. Realización de las actividades para casa con el ordenador \\
\hline $\mathrm{R}_{1}$ & 4. Utilización de Internet de formas muy distintas para hacer cosas que me interesan \\
$\mathrm{R}_{2}$ & 5. Uso Internet para hacer cosas, aunque no se pida que se hagan con ello \\
\hline $\mathrm{R}_{3}$ & 6. Uso el ordenador para hacer crear cosas nuevas \\
$\mathrm{P}_{1}$ & 7. En la escuela, las tareas que se mandan se realizan mejor con un ordenador \\
$\mathrm{P}_{2}$ & 8. En clase, existe la necesidad de usar el ordenador para trabajar, incluso cuando el profesor no plantea \\
$\mathrm{R}_{4}$ & 9. Podría usar el ordenador para hacer tareas de clase de manera más fácil, aunque nadie me enseñe \\
$\mathrm{P}_{3}$ & 10. Aprendizaje de cosas en la escuela que ahora no podría realizar sin ordenador \\
$\mathrm{R}_{5}$ & 11. Sabría utilizar el ordenador e Internet para aprender mucho más de lo que aprendo en la escuela \\
\hline $\mathrm{D}$
\end{tabular}

D: Dominio; P: Preferencia; R: Reintegración

La segunda escala, Emociones asociadas al uso de las TIC, se materializa a través de 9 ítems tal como se muestra en la tabla 2. Como puede observarse, se han incluido las emociones en su formulación positiva para una mayor claridad y facilidad de comprensión del instrumento por parte de los estudiantes. No obstante, se ha añadido un ítem final (inverted item) en su formulación negativa para contrastar un posible posicionamiento positivo de los resultados.

\section{TABLA 2. ESCALA SOBRE EL ESTADO EMOCIONAL ASOCIADO AL USO DE LAS TIC}

1. Curiosidad con las cosas nuevas que aprendo

2. Más apoyo por parte de mis profesores

3. Orgullo por las cosas que hago

4. No me siento frustrado, me sale todo

5. Mayor confianza en mí (autoestima)

6. Mayor seguridad, no me pongo tan nervioso/a

7. Diversión, me aburro menos con las cosas que hacemos

8. Bienestar y satisfacción

9. Si no me permitieran usar tecnologías, me sentiría muy insatisfecho/a

Para dar respuesta a nuestros objetivos científicos se han aplicado diferentes técnicas estadísticas de análisis cuantitativo mediante el paquete estadístico SPSS v.24.

De cara a responder a los propósitos del estudio que aquí se presenta y establecer el modelo predictivo, la técnica utilizada ha sido la regresión logística binaria. Técnica que resulta idónea para modelar cómo influye en la probabilidad de un suceso, en este caso la generación de emociones positivas o negativas en los 
estudiantes, la presencia o no de los usos TIC llevados a cabo por ellos. De este modo, es posible establecer una sistematización jerárquica de la capacidad predictiva de las diferentes variables estudiadas con respecto al estado emocional.

Los coeficientes de regresión logística determinados para las variables del modelo se han utilizado para la estimación de las odds ratio. Éstas constituyen parámetros de cuantificación de probabilidad, que indican cuánto más probable es la aparición de estados emocionales positivos vinculada a los usos que hacen los estudiantes de las TIC.

Para comprobar la capacidad predictiva del Uso de las TIC sobre la dimensión afectiva, se ha creado una nueva variable (índice) representando el valor medio de los elementos de la escala Emociones asociado al uso de las TIC. Finalmente, este índice se convierte en una variable dummy creando un punto intermedio en los valores obtenidos siguiendo el criterio que se detalla a continuación:

- Intervalo de 1 a 2,99 = 0 (Presentan Emociones negativas)

- Intervalo de 3 a 5 = 1 (Presentan Emociones positivas).

\section{RESULTADOS}

En cuanto al análisis de la consistencia interna de las escalas, la fiabilidad se ha realizado mediante el alfa de Cronbach que arroja un coeficiente de ,808 y ,840 para las dos escalas utilizadas tal como se muestra en la tabla 3. Los valores obtenidos nos indican, por tanto, que las escalas elaboradas presentan una aceptable consistencia interna y fiabilidad.

\begin{tabular}{lc}
\hline \multicolumn{2}{c}{ TABLA 3. ALFA DE CRONBACH DE LA ESCALA } \\
\hline \multicolumn{1}{c}{ Escala } & Alpha de Cronbach \\
\hline Usos de las TIC &, 808 \\
\hline Estado Emocional asociado a las TIC &, 840 \\
\hline
\end{tabular}

La técnica estadística utilizada para el análisis de la estructura que subyace al instrumento ha sido el Análisis Factorial Clásico, utilizando el procedimiento de componentes principales (AFCP). Analizando la significatividad o no de las relaciones entre variables, comprobamos (tabla 4), como ambos resultados nos indican la adecuación del análisis factorial a los datos.

\begin{tabular}{lcc}
\hline \multicolumn{4}{c}{ TABLA 4. PRUEBA DE KMO Y BARLETT } \\
\hline \multicolumn{1}{c}{ Escala } & Medida Kaiser-Meyer-Olkin & Prueba de esfericidad de Barlett \\
\hline Usos de las TIC &, 893 & Sig. , 000 \\
\hline Estado Emocional asociado a las TIC &, 866 & Sig. , 000 \\
\hline
\end{tabular}


Por un lado, y teniendo en cuenta el criterio mayor de 0,60 , podemos considerar el valor KMO significativo. De la misma forma, consideramos significativa la matriz de correlaciones mediante la prueba de esfericidad de Barlett $(\mathrm{p} \leq 0.05)$. Del mismo modo, la comunalidad de todos los ítems es superior a $0,4 \mathrm{y}$, por tanto, podemos afirmar que éstos están relacionados entre sí.

\begin{tabular}{cccc}
\hline & \multicolumn{3}{c}{ TABLA 5. VARIANZA TOTAL EXPliCADA: USOS DE LAS TIC } \\
\cline { 2 - 4 } Componente & Autovalores Iniciales \\
& Total & \% DE VARIANZA & \% ACumulado \\
\hline 1 & 4,273 & 38,847 & 38,847 \\
\hline 2 & 1,038 & 9,440 & 48,287 \\
\hline 3 & 1,022 & 9,292 & 57,579 \\
\hline
\end{tabular}

Del análisis de la matriz de componentes rotados (VARIMAX) de la primera escala, se extraen tres factores cuyo autovalor es superior a 1 (tabla 5) y que explican el 57,58\% de la varianza. Los tres factores obtenidos los hemos denominado «Reintegración», con un autovalor de 4,273 y saturado por los ítems 4, 5, 6, 9 y 11; "Dominio", con un autovalor de 1,038 y saturado por los ítems 1,2 y 3 ; «Preferencia» con autovalor 1,022 y saturado por los ítems 7,8 y 10 respectivamente.

\begin{tabular}{cccc}
\hline \multirow{2}{*}{ TABLA 6. VARIANZA TOTAL EXPLICADA: ESTADO EMOCIONAL ASOCIADO AL USO DE LAS TIC } \\
\hline \multirow{3}{*}{ COMPONENTE } & TotAL & \% DE VARIANALORES INICIALES & \% ACUMULADO \\
\cline { 2 - 4 } & 3,637 & 40,410 & 40,410 \\
\hline 1 & 1,110 & 12,338 & 52,748 \\
\hline 2 & & & \\
\hline
\end{tabular}

En cuanto a la segunda escala (tabla 6), los dos factores obtenidos los hemos denominado "Estado emocional espontáneo», con un autovalor de 3,637 y saturado por los ítems 1, 2, 3, 5 y 8; «Estado emocional valorado», con un autovalor de 1,110 y saturado por los ítems 4, 6, 7 y 9 respectivamente. La obtención de estos dos factores en las emociones se sustenta en hallazgos de la neurociencia (Reyes, Conde \& Colás, 2017). En un estudio de Marcovich (2011) se concluye que existen dos tipos de aprendizaje: un aprendizaje emocional, relacionado con la parte reptil del cerebro, y que, en palabras de la autora, es rápido, indisoluble e involuntario. Por lo tanto, el primer factor recogería las emociones que se producen cuando se usan las TIC y se generan de forma automática emociones primitivas en los estudiantes, tales como Curiosidad (Ítem), Apoyo (Ítem 2), Orgullo (Ítem 3), Confianza (Ítem 5) y Bienestar-Satisfacción (Ítem 8). Por otra parte, nos encontramos con un apren- 
dizaje que origina una respuesta emocional más elaborada. Según la autora, este aprendizaje, de naturaleza cognitivo-ejecutiva, también requiere de una valoración e interpretación de las emociones instintivas (recogidas en el primer factor). De este modo, se puede considerar que las emociones que integran este segundo factor están soportadas en emociones previas de carácter más instintivo y espontáneo. En este bloque se situarían la Frustración (Ítem 4), la Seguridad (Ítem 6), la Diversión (Ítem 7) y el inverted item (Ítem 9).

El modelo de regresión se ha construido siguiendo un método de introducción por pasos. Las siete variables obtenidas y que, por tanto, forman parte de la ecuación de regresión se recogen en la tabla 7. Todas las variables obtenidas son significativas de acuerdo con el estadístico Chi-Cuadrado de Wald, cuyo valor observado permite rechazar la hipótesis nula para un $\mathrm{p}$ valor $<0,05$. Se ha decidido mantener el ítem «Podría usar el ordenador para hacer tareas de clase de manera más fácil, aunque nadie me enseñe» por encontrarse próximo al límite, asumiendo un $\mathrm{p}$ valor $<0,06$. Por tanto, se decide eliminar de la escala inicial propuesta los ítems D3, R1, R2 y P1 en tanto no presentan valor predictivo relevante.

\begin{tabular}{|c|c|c|c|c|c|}
\hline \multicolumn{6}{|c|}{$\begin{array}{l}\text { TABLA 7. PRUEBAS DE SIGNIFICACIÓN PARA LOS COEFICIENTES } \\
\text { DE LAS VARIABLES INCLUIDAS EN LA ECUACIÓN }\end{array}$} \\
\hline Uso dE LAS TIC & B & $\begin{array}{l}\text { ERROR } \\
\text { TÍPICO }\end{array}$ & WALD & GL & SIG. \\
\hline $\begin{array}{l}\text { Realización, sin problema, de las actividades } \\
\text { escolares con el ordenador }\end{array}$ &, 139 &, 056 & 6,188 & 1 & ,013 \\
\hline $\begin{array}{l}\text { Conocimiento del funcionamiento de las tecno- } \\
\text { logías, y su uso para hacer los deberes }\end{array}$ &, 181 &, 051 & 12,753 & 1 &, 000 \\
\hline $\begin{array}{l}\text { Uso del ordenador para crear cosas nuevas, que } \\
\text { nadie me ha enseñado }\end{array}$ &, 232 &, 044 & 28,200 & 1 &, 000 \\
\hline $\begin{array}{l}\text { En clase, existe la necesidad de usar el ordenador } \\
\text { para trabajar, incluso cuando el profesor no plan- } \\
\text { tea la actividad a través de este medio }\end{array}$ &, 113 &, 049 & 5,445 & 1 &, 020 \\
\hline $\begin{array}{l}\text { Podría usar el ordenador para hacer tareas de clase } \\
\text { de manera más fácil, aunque nadie me enseñe }\end{array}$ & ,096 &, 051 & 3,549 & 1 &, 060 \\
\hline $\begin{array}{l}\text { Aprendizaje de cosas en la escuela que ahora no } \\
\text { podría realizar sin ordenador en el colegio }\end{array}$ & ,244 &, 046 & 28,391 & 1 &, 000 \\
\hline $\begin{array}{l}\text { Sabría utilizar el ordenador e Internet para apren- } \\
\text { der mucho más de lo que aprendo en la escuela }\end{array}$ & ,257 &, 051 & 25,891 & 1 &, 000 \\
\hline Constante & $-3,083$ & 260 & 140,388 & 1 & ,000 \\
\hline
\end{tabular}

La bondad del modelo obtenido se ha contrastado utilizando diferentes pruebas estadísticas. La prueba ómnibus presenta una significación del ,000 y esto indica que el ajuste entre las variables contempladas permite la generación de un modelo con capacidad predictiva. 


\begin{tabular}{ccc}
\hline \multicolumn{3}{c}{ TABLA 8. PRUEBA ÓMNIBUS DE COEFICIENTES DE MODELO } \\
\hline Chi-CuAdrado & gl & Sig. \\
\hline 356,756 & 7 &, 000 \\
\hline
\end{tabular}

De igual modo, se puede afirmar la calidad predictiva de nuestro modelo en tanto es capaz de explicar entre un $20 \%$ y un $28 \%$ de la varianza de la variable dependiente tal como se observa en la tabla 9.

\begin{tabular}{cc}
\hline \multicolumn{2}{c}{ TABLA 9. RESUMEN DEL MODELO } \\
\hline R CUADRADO DE COX Y SNELL & R CUADRADO DE NAGELKERKE \\
\hline 200 &, 285 \\
\hline
\end{tabular}

Además, se ha sometido el modelo a contraste mediante la prueba de Hosmer y Lemeshow, arrojando un estadístico Chi-Cuadrado de 9,977, con una probabilidad asociada $\mathrm{p}=0,267$. Esta prueba parte de la idea de que, si el ajuste es bueno, un valor alto de la probabilidad predicha $(\mathrm{p})$ se asociará con el resultado 1 de la variable binomial dependiente.

\begin{tabular}{ccc}
\hline \multicolumn{3}{c}{ TABLA 10. PRUEBA DE HOSMER Y LEMESHOW } \\
\hline Chi-CuAdrado & GL & Sig. \\
\hline 9,977 & 8 &, 267 \\
\hline
\end{tabular}

En cuanto a la capacidad predictiva o validez del modelo, se ha conseguido la clasificación correcta de un $76,5 \%$ de los sujetos (tabla 11), mostrando una alta especificidad del $90,2 \%$ y una sensibilidad media del $43,6 \%$. Dicho de otro modo, el modelo es capaz de pronosticar de manera acertada al menos en tres de cada cuatro sujetos estudiados.

\begin{tabular}{|c|c|c|c|c|}
\hline \multicolumn{5}{|c|}{ TABLA 11. MATRIZ DE CLASIFICACIÓN } \\
\hline \multirow{3}{*}{ Observado } & & \multicolumn{3}{|c|}{$\begin{array}{l}\text { Pronosticado } \\
\end{array}$} \\
\hline & & \multicolumn{2}{|c|}{ EMOCiONES Positivas } & \multirow{2}{*}{$\%$ ACUMULADO } \\
\hline & & No & Sí & \\
\hline \multirow{2}{*}{ Emociones Positivas } & No & 204 & 264 & 43,6 \\
\hline & Sí & 111 & 1018 & 90,2 \\
\hline Porcentaje Global & & & & 76,5 \\
\hline
\end{tabular}

Esta predicción aumenta significativamente en aquellos estudiantes que manifiestan emociones positivas, siendo posible predecir el 90,2\% de estos casos. Es decir, para al menos nueve de cada diez estudiantes, ha sido posible pronosticarlo. 


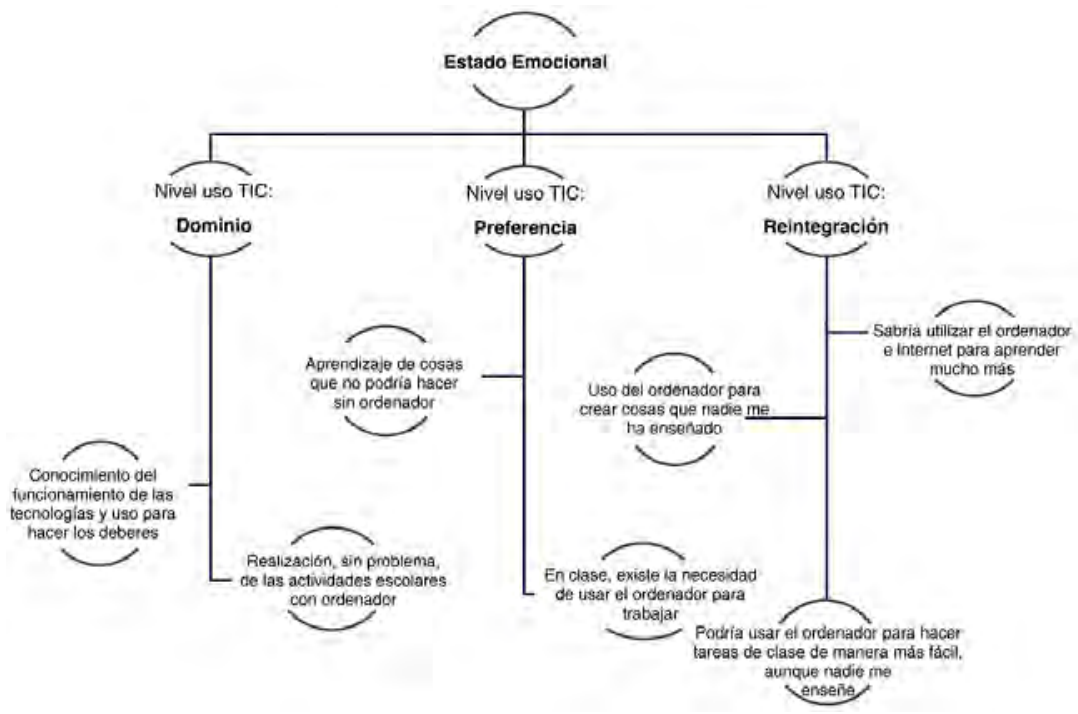

Figura 6. Variables Jerarquizadas según su potencia predictora y su peso odds Ratio.

Una vez confirmada la bondad de ajuste del modelo, estudiamos las variables incluidas en el mismo. Tal como se observa en la tabla 7, todas las variables muestran un coeficiente de regresión positivo y, por tanto, podemos afirmar que las variables introducidas son facilitadoras o predictoras de estados emocionales positivos. Sumado a lo anterior, se toman en consideración las odds ratio, que representan un modo de cuantificar cuánto más probable es el suceso cuando los factores predictores incrementan su valor. En la tabla 12 mostramos las odds ratio para cada variable del modelo y los límites de su intervalo de confianza con un nivel del 95\%.

\begin{tabular}{|c|c|c|c|}
\hline \multicolumn{4}{|c|}{ TABLA 12. ODDS RATIO E INTERVALOS DE CONFIANZA } \\
\hline \multirow{3}{*}{ Uso dE LAS TIC } & \multirow{3}{*}{ OdDS Ratio } & \multirow{2}{*}{\multicolumn{2}{|c|}{$\begin{array}{c}\text { Intervalo de Confianza } \\
95 \% \text { PaRa ODDS Ratio }\end{array}$}} \\
\hline & & & \\
\hline & & INFERIOR & SUPERIOR \\
\hline $\begin{array}{l}\text { Realización, sin problema, de las actividades escolares con } \\
\text { el ordenador }\end{array}$ & 1,149 & 1,030 & 1,282 \\
\hline $\begin{array}{l}\text { Conocimiento del funcionamiento de las tecnologías, y su } \\
\text { uso para hacer los deberes }\end{array}$ & 1,198 & 1,085 & 1,324 \\
\hline $\begin{array}{l}\text { Uso del ordenador para crear cosas nuevas, que nadie me } \\
\text { ha enseñado }\end{array}$ & 1,261 & 1,158 & 1,374 \\
\hline $\begin{array}{l}\text { En clase, existe la necesidad de usar el ordenador para } \\
\text { trabajar, incluso cuando el profesor no plantea la actividad } \\
\text { a través de este medio }\end{array}$ & 1,120 & 1,018 & 1,232 \\
\hline
\end{tabular}




\begin{tabular}{lccc}
\hline $\begin{array}{l}\text { Podría usar el ordenador para hacer tareas de clase de manera } \\
\text { más fácil, aunque nadie me enseñe }\end{array}$ & 1,100 &, 996 & 1,216 \\
\hline $\begin{array}{l}\text { Aprendizaje de cosas en la escuela que ahora no podría } \\
\text { realizar sin ordenador en el colegio }\end{array}$ & 1,277 & 1,167 & 1,397 \\
\hline $\begin{array}{l}\text { Sabría utilizar el ordenador e Internet para aprender mucho } \\
\text { más de lo que aprendo en la escuela }\end{array}$ & 1,294 & 1,171 & 1,428 \\
\hline \begin{tabular}{l} 
Constante \\
\hline
\end{tabular} &, 046 & & \\
\hline
\end{tabular}

Basándonos en las odds ratio, a continuación (figura 5), se muestra una clasificación de los factores incluidos en el modelo estableciendo una jerarquía de acuerdo a su potencia predictiva.

En cuanto a los resultados obtenidos en el modelo predictivo, se comprueba como los usos relacionados con el constructo Reintegración son los que mayor peso presentan y por tanto son los que mayor influencia tienen a la hora de generar estados emocionales positivos. En concreto, el uso de las TIC para aprender y crear cosas nuevas se desmarcan como los que más emociones positivas producen. En cuanto a la Preferencia, la preferencia de la aplicación del ordenador como herramienta de aprendizaje es otro elemento que configura la aparición de los estados emocionales positivos en los estudiantes. Por último, los usos relacionados con el Dominio, aunque también se relacionan favorablemente con niveles positivos de la dimensión afectiva, no tienen el mismo protagonismo que las categorías anteriores.

\section{CONCLUSIONES}

De los resultados obtenidos en el procedimiento de diseño y análisis de las propiedades psicométricas de la escala, se extraen algunas conclusiones y propuestas. En primer lugar, consideramos que nuestro estudio presenta un instrumento válido y fiable, capaz de ser útil tanto en el marco de este estudio como aplicable a otros contextos, para cubrir los objetivos propuestos. Por tanto, podemos confirmar que la escala que se propone puede ser una herramienta útil para ser usada por el profesorado debido a su carácter predictivo y evaluativo, de cara a desarrollar propuestas TIC en el aula mediante la evaluación del nivel de estado emocional de los alumnos y los usos asociados al nivel de competencia digital.

En segundo lugar, se observa como los factores obtenidos de manera empírica respaldan los supuestos teóricos, los cuales afirman que el uso de la TIC en el aprendizaje tiene un impacto positivo en el estado emocional de los estudiantes. En este sentido, de manera general, los hallazgos confirman que los estudiantes presentan niveles más altos de emociones positivas cuando los usos y aplicaciones de las TIC son más complejos. Tal como se ha visto, aquellos estudiantes que favorecen los usos de Reintegración y Preferencia frente a los de Dominio desarrollan estados emocionales más positivos. Del mismo modo, en el modelo predictivo obtenido, se observa que los estudiantes que presentan una gama más alta de aprovechamiento de los recursos tecnológicos disponibles en la escuela (conocimiento de las tecnologías, 
realización de tareas con el ordenador, etc.) presentan estados emocionales activos, positivos y de bienestar personal.

Estos resultados convergen con investigaciones centradas en el pensamiento computacional, que señalan que cuando los estudiantes son agentes activos y creadores de sus propios contenidos, tienen una mejor predisposición hacia el aprendizaje y presentan mejor estado emocional (Román, 2016). Asimismo, en esta misma línea, estudios recientes destacan como el desarrollo de habilidades cognitivas como el aprendizaje autónomo o la creatividad, que se enmarcaría dentro del constructo de la Reintegración, se relaciona directamente con la motivación y el engagement del alumnado (Carlson, 2005; Reyes, 2016; Román, 2016). Estos estudios han puesto sobre la mesa el hecho de que los estudiantes se sienten más motivados y conectados con sus estudios cuando éstos incorporan aspectos multimedia y TIC. En otras palabras, contenidos que estén expresados más acorde con sus discursos culturales y conectados con sus intereses intelectuales. Por tanto, la educación del presente y del futuro debe aceptar la multitarea como parte, ya, de la vida cotidiana de los estudiantes y, por tanto, aprender a utilizarla para lograr una educación efectiva, de calidad y generadora de estados emocionales positivos. Por tanto, mientras seamos capaces de ofrecer a nuestros estudiantes contextos educativos enriquecidos con múltiples estímulos y estrategias multimodales, en los que asuman un rol activo, tendremos alumnos con mejor estado emocional asociado a los usos de las TIC (Barnes, Desmarais, Romero \& Ventura, 2009, Reyes, 2016).

Por último, de forma prospectiva y para previsión de futuras investigaciones consideramos que el enfoque utilizado, basado en la práctica que se lleva a cabo en las aulas, así como el modelo y su capacidad predictiva, pueden dar lugar a instrumentos o intervenciones en el aula capaces de detectar los estados emocionales de los estudiantes. Esto nos va a permitir mejorar y/o mantener los estados emocionales positivos y, en aquellos casos que presenten emociones negativas, favorecer, alentar y/o volver a conectarlos emocionalmente a través del uso y desarrollo de actividades que impliquen las TIC, mejorando así sus niveles de alfabetización digital. 


\section{REFERENCIAS BIBLIOGRÁFICAS}

Area, M. y Ribeiro, T. (2012). «De lo sólido a lo líquido: Las nuevas alfabetizaciones ante los cambios culturales de la Web 2.0». Comunicar, XIX (38), 13-20.

Arguedas, M., Daradoumis, T. y Xhafa, F. (2016). «Analyzing How Emotion Awareness Influences Students' Motivation, Engagement, Self-Regulation and Learning Outcome». Educational Technology \& Society, 19 (2), 87-103.

Arnold, J. (2000). La dimensión afectiva en el aprendizaje de idiomas. Madrid, España: Cambridge University Press.

Aviram, A. y Eshet-Alkalai, Y. (2006). «Towards a theory of digital literacy: three scenarios for the next steps». European Journal of Open, Distance and E-Learning, 1, 1-11.

Barnes, T., Desmarais, M., Romero, C. y Ventura, S. (2009). Educational Data Mining 2009: $2^{\text {nd }}$ International Conference on Educational Data Mining Proceedings. Córdoba, España: Universidad de Córdoba.

Beaudry, A. y Pinsonneault, A. (2010). «The Other Side of Acceptance: Studying the Direct and Indirect Effects of Emotions on Information Technology Use». MIS Quarterly, 34 (4), 689-710.

BeLLI, S. y GiL-JuÁrez, A. (2011). «Llorar delante de una pantalla plana. Emociones compartidas en un locutorio». Barataria. Revista Castellano-Manchega de Ciencias Sociales, (12), 137-150.

Belli, S., Harré, R. y IÑiguez, L. (2009). «Emotions in Technoscience The performance of velocity. Human Affairs». In C. Peter, L. Axelrod, S. Afzal, H. Agius, E. Crane \& M. Balaam (coord), Workshop proceedings emotion in HCI-Real World Challenge (pp. 6-13). Cambridge, England: University of Cambrdige.

Bhandari, U. y Chang, K. (2014). «Role of emotions and aesthetics in ICT usage for underserved communities: a NeuroIS investigation. Research in Progress". In the Thirty Fifth International Conference on Information Systems (pp. 1-12). Auckland, New Zealand: ICIS.

BI, L. y FAN, X. (2011). «Emotion recognition from EEG based on Bayesian networks». Energy Procedia, 11, 278-285.

Butz, N.T., Stupnisky, R.H. y Pekrun, R. (2015). «Students' emotions for achievement and technology use in synchronous hybrid graduate programmes: a control-value approach». Research in Learning Technology, 23, 1-16.

Carlson, S. (2005). «The net generation goes to college». The chronicle of Higher Education, 52 (7), 34.

Charitaki, G. (2015). «The effect of ict on emotional education and development of young children with autism spectrum disorder». Procedia Computer Science, 65, 285-293.

Chen, C. y Lee, T. (2011). «Emotion recognition and communication for reducing second-language speaking anxiety in a web-based one-to-one synchronous learning environment». British Journal of Educational Technology, 42 (3), 417-440.

Chen, C. y Sun, Y. (2012). «Assessing the effects of different multimedia materials on emotions and learning performance for visual and verbal style learners». Computers \& Education, 59 (4), 1273-1285.

Chen, C. y WANG, H. (2011). «Using emotion recognition technology to assess the effects of different multimedia materials on learning emotion and performance». Library \& Information Science Research, 33 (3), 244-255. 
Colás, P. (2006). «Metodología pedagógica para e-learning desde un enfoque sociocultural». En VI Conferencia Internacional sobre E-learning y Tecnologías de Educación. Lisboa, Portugal.

Colás, P., Conde, J. y GonzÁlez, T. (2015). «Evaluación de políticas TIC: competencias digitales». EDUSK. Revista monográfica de Educación, 4-Calidad educativa: avances, aportaciones y retos, 289-329. São Paulo: editorial skepsis +.

Colás, P., Rodríguez, M. y Jiménez, R. (2005). «Evaluación de e-learning. Indicadores de calidad desde el enfoque sociocultural». Teoría de la Educación. Educación y Cultura en la Sociedad de la Información, 6 (2), online. Recuperado el 6/04/2017, de http://campus.usal.es/-teoriaeducacion/rev_numero_06_2/n6_02art_colas_rodriguez_jimenez.htm.

Comisión Europea (2006). «Recommendation of the European Parliament and of the Council of 18 December 2006 on key competences for lifelong learning (2006/962/EC)». Official Journal of the European Union, L 394, 10-18.

Comisión Europea (2010). «Comunicación “Agenda digital para Europa” [COM (2010) 245 final - no publicada en el Diario Oficial]». Brussels: European Commission.

Comisión Europea (2014). «Chapter 4. Digital Competences in the Digital Agenda». En Comisión Europea (ed.), Digital for Europe (pp. 95-121). Brussels: European Commission.

Conde, J. (2017). La mediación de las TIC en la creación de ambientes de aprendizaje y el logro de competencias digitales. Tesis doctoral. Sevilla, España: Universidad de Sevilla.

De Lera, E., Fernández, C. y Almirall, M. (2009). «Emotions: the forgotten key success in online learning». In $13^{\text {th }}$ International Conference and World Bank-KERIS High Level Seminar on ICT in Education. Hangzhou, China: UNESCO-APEID.

De Pablos, J. (2003). «La tecnología educativa hoy no es como ayer: nuevos enfoques, nuevas miradas». Tecnología y Comunicación Educativas (TyCE), 37 (1), 5-21.

De Pablos, J. Rebollo, M. y Aires, L. (1999) «Para un estudio de las aportaciones de Mijaíl Bajtín a la Teoría Sociocultural». Una aproximación Educativa. Revista de Educación, (320), 223-253.

De Pablos, J., Colás, P., González, T. y Camacho, C. (2013). «Teacher Well-being and Innovation with Information and Communication Technologies; Proposal for a structural model». Quality \& Quantity, 47 (5), 2755-2767.

De Pablos, J., Colás, P., Conde, J. y Reyes, S. (2017). «La competencia digital de los estudiantes de educación no universitaria: variables predictivas». Bordón. Revista de Pedagogía, 69 (1), 169-185.

Eow, Y., Wan-ZaH, W., Rosnaini, M. y Roselan, B. (2010). «Appreciative Learning Approach: A New Pedagogical Option». In T. Hirachima, A.F. Mohd, L.F. Kwok, S.L. Wong, S.C. Kong \& F.Y. Yu (eds.), Proceedings of the $18^{\text {th }}$ International Conference on Computers in Education (pp. 607-614). Putrajaya, Malaysia: Asia-Pacific Society for Computers in Education.

García, S., Garrote, D. y Jiménez, S. (2016). «Uso de las TIC en el Trastorno de Espectro Autista: aplicaciones». edmetic. Revista de Educación Mediática y TIC, 5 (2), 134-157.

Hardy, C., Ogden, J., Newman, J. y Cooper, S. (2002). Autism and ICT: A guide for teachers and parents. London, England: David Fulton.

Huertas, A. y Pantoja, A. (2016). «Efectos de un programa educativo basado en el uso de las TIC sobre el rendimiento académico y la motivación del alumnado en la asignatura de tecnología de educación secundaria». Educación XX1, 19 (2), 229-250. 
IlomäKi, L., Kantosalo, A. y Lakkala, M. (2011). What is digital competence? Brussels: European Schoolnet.

Johnson, L., Adams Becker, S., Estrada, V. y Freeman, A. (2014). NMC horizon report: 2014 Higher Education edition. Austin, Texas: The New Media Consortium.

Kaiser Family Foundation. (2010). Generation M2: Media in the Lives of 8-to 18-year-old. Kaiser Family Foundation.

Kwah, H., Milne, C., Tsai, T., Goldman, R. y Plass, J.L. (2016). «Emotional engagement, social interactions, and the development of an afterschool game design curriculum». Cultural Studies of Science Education, 11 (3), 713-740.

Lehman, B., D’Mello, S. y Graesser, A. (2012). «Confusion and complex learning during interactions with computer learning environments». The Internet and Higher Education, 15 (3), 184-194.

Losada, D., Correa, J. y Fernández, L. (2017). «El impacto del modelo “un ordenador por niño" en la Educación Primaria: Un estudio de caso». Educación XX1, 20 (1), 339-361.

Lozano, J., Ballesta, J. y Alcaraz, S. (2011). «Software for Teaching Emotions to Students with Autism Spectrum Disorder». Comunicar, 36, 139-148.

Marchand, G. y Gutierrez, A. (2012). «The role of emotion in the learning process: comparisons between online and face-to-face learning settings». The Internet and Higher Education, 15 (3), 150-160.

Marcovich, A. (2011). "Cerebro y Aprendizaje. Factores obstaculizadores y favorecedores para el aprendizaje». En Asociación Educar (coord.), Encuentro sobre Ciencias y Neurociencias aplicadas al desarrollo humano. Recuperado el 5/7/2017, de https://goo.gl/v5XATD.

Martin, A. y Grudziecki, J. (2006). «DigEuLit: Concepts and tools for digital literacy development». ITALICS: Innovations in Teaching \& Learning in Information \& Computer Sciences, 5 (4), 246-264.

MÉndeZ, D. (2015). «Estudio de las motivaciones de los estudiantes de secundaria de física y química y la influencia de las metodologías de enseñanza en su interés». Educación XX1, 18 (2), 215-235.

Moore, D. y TAYLOR, J. (2000). «Interactive multimedia systems for people with autism». Journal of Educational Media, 2 (3), 169-177.

Nummenmaa, M. (2007). Emotions in a web-based learning environment. Dissertation of University of Turku, Finland. Eduational Technology Unit.

OECD (2015). Students, Computers and Learning: Making the Connection. Bruselas: PISA, OECD. Recuperado el 6/03/2017, de http://dx.doi.org/10.1787/9789264239555-en.

Olitsky, S. y Milne, C. (2012). «Understanding engagement in science education: The psychological and the social». In B. Fraser, K. Tobin, \& C. McRobbie (eds.), Second international handbook of science education (pp. 19-33). Dordrecht, Netherlands: Springer.

Parboteeah, D., Valacich, J. y Wells, J. (2009). «The Influence of Website characteristics on a Consumer's Urge to Buy Impulsively». Information Systems Research, 20 (1), 60-81.

Park, B., Plass, J.L. y Brünken, R. (2014). «Cognitive and affective processes in multimedia learning». Learning and Instruction, 29, 125-127.

Reyes, S. (2016). Fortalecer la implicación y el compromiso de los estudiantes con la universidad. Una visión multidimensional del engagement. Tesis doctoral. Sevilla, España: Universidad de Sevilla. 
Reyes, S., Conde, J. y Colás, P. (2017). «Validación confirmatoria de una escala para medir las emociones asociadas al uso de las TIC en contextos educativos». Comunicación en XXVJornadas Universitarias de Tecnología Educativa. JUTE. Burgos, España.

Rodríguez, N. (2016). «¿Las TIC como mediadoras en la enseñanza-aprendizaje de lenguas extranjeras?». Opción, 32 (10), 569-588.

Román, M. (2016). Codigoalfabetización y pensamiento computacional en educación primaria y secundaria: validación de un instrumento y evaluación de programas. Tesis doctoral. Madrid, España: Universidad Nacional de Educación a Distancia. UNED.

Schmidt, J., Kackar-Cam, H., Strati, A. y Shumow, L. (2015). «The Role of Challenge in Students' Engagement and Competence in High School Science Classrooms: Hispanic and Non-Hispanic Whites Compared». NCSSS Journal, 20 (1), 20-26.

Senret, I. y Kommers, P. (2014). «Conceptual issuses of the digital competence development in the framework of the Council of the European Union». In M. Turčáni, M. Drlík, J. Kapusta \& P. Švec (ed.), DIVAI 2014 - Distance Learning in Applied Informatics. Conference Proceedings (s.p.). Nitra (Slovakia): Faculty of Natural Sciences, Department of Informatics.

Senkbeil, M., Ihme, J.M. y Wittwer, J. (2013). «The test of technological and information literacy (TILT) in the national educational panel study: Development, empirical testing, and evidence for validity». Journal für Bildungsforschung Online, 5 (2), 131-169.

Serrano, J. (2015). «Emociones en el uso de la tecnología: un análisis de las investigaciones sobre teléfonos móviles». Observatorio $\left(O B S^{*}\right), 9(4), 101-112$.

Shen, L., WANG, M. y Shen, R. (2009). «Affective e-learning: using «emotional» data to improve learning in pervasive learning environment». Educational Technology \& Society, 12 (2), 176-189.

Suryani, A., Subali, E. y Ratu, A. (2016). Proceedings of the Third International Seminar of PRASASTI. Current Research in Linguistics (pp. 80-91). Surakarta, Indonesië: Universitas Sebelas Maret.

Terrazas, T., SÁnchez, S. y Becerra, M. (2016). «Las TIC como herramienta de apoyo para personas con Trastorno del Espectro Autista (TEA)». Revista de Educación Inclusiva, 9 (2), 102-136.

Thong, J., Hong, S. y TAm, K. (2006). «The Effects of Post-Adoption Beliefs on the Expectation-Confirmation Model for Information Technology Continuance». International Journal of Human-Computer Studies, 64 (9), 799-810.

Um, E., Plass, J., Hayward, E. y Homer, B. (2011). Emotional design in multimedia learning. Journal of Educational Psychology, 104 (2), 485-498.

Valverde, J., Fernández, M. y Revuelta, F. (2013). «El bienestar subjetivo ante las buenas prácticas educativas con TIC: su influencia en profesorado innovador». Educación XX1, 16 (1), 255-280.

van der Heijden, H. (2004). «User Acceptance of Hedonic Information Systems». MIS Quarterly, $28(4), 695-704$.

van Deursen, A. y van Dijk, J. (2009). «Using the internet: skill related problems in users' online behavior». Interacting with computers, 21 (6), 393-402.

Venkatesh, V. y Bala, H. (2008). «Technology Acceptance Model 3 and a Research Agenda on Interventions». Decision Sciences, 39 (2), 273-315.

WANG, M. (2014). «The current practice of integration of information communication technology to English teaching and the emotions involved in blended learning». TOJET: The Turkish Online Journal of Educational Technology, 13 (3), 188-201. 
Wilson, M., Scalise, K. y Gochyyev, P. (2015). «Rethinking ICT literacy: From computer skills to social network settings». Thinking Skills and Creativity, 18, 65-80.

ZHANG, P. (2013). «The affective response model: A theoretical framework of affective concepts and their relationships in the ict context». MIS quarterly, 37(1), 247-274.

ZHONG, Z. (2011). «From access to usage: The divide of self-reported digital skills among adolescents». Computers \& Education, 56 (3), 736-746.

Zhou, M. (2013). "I am Really Good at It" or "I am Just Feeling Lucky": the effects of emotions on information problem-solvin». Educational Technology Research and Development, 61 (3), 505-520.

Zylka, J., Christoph, G., Kroehne, U., Hartig, J. y Goldhammer, F. (2015). «Moving beyond cognitive elements of ICT literacy: First evidence on the structure of ICT engagement». Computers in Human Behavior, 53, 149-160. 